\title{
Volume 13 Referees
}

c) Konrad Lorenz Institute for Evolution and Cognition Research 2018

We wish to thank the following scholars who have generously shared their time and expertise to serve as reviewers for Biological Theory over the past year. We are truly grateful for their help, as well as for the assistance from reviewers who chose to remain anonymous.

Ramsey Affifi

Mahesh Ananth

Michael Anderson

Marc Artiga

Claudia Bank

Leonardo Bich

John Bickle

Doris Bucher

Brett Calcott

Paco Calvo

Matthew Cobb

Charbel El-Hani

G. Bard Ermentrout

Paul Ewald

Agustín Fuentes
Eric Funkhouser

Arran Gare

Richard Gawne

Root Gorelick

Peter Graham

Benedikt Hallgrimson

Michal Hladky

Ema Hrouzkova

Eugene Koonin

Ehud Lamm

Marta Linde-Medina

David S. Moore

Lenny Moss

Vidyanand Nanjundiah

Lennart Nordenfelt
Samir Okasha

Manus Patten

Gualtiero Piccinini

Ronald Planer

William Ratcliff

Matt Rossano

Isabella Sarto-Jackson

Michael Sheehan

Chris Sinha

Kelly Smith

Kim Sterelny

Eörs Szathmary

James Tabery 